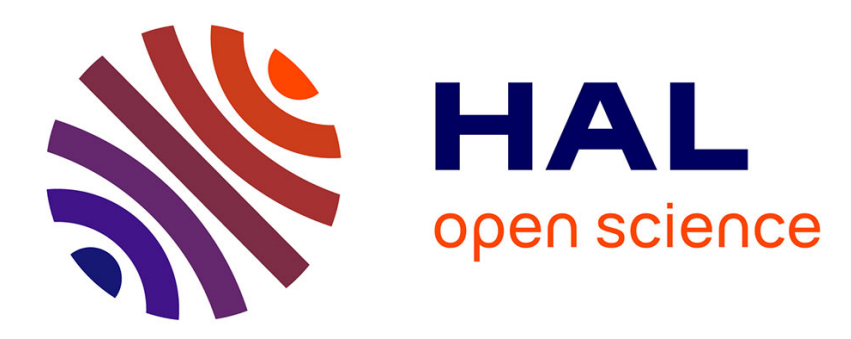

\title{
MODULATIONAL POLARIZATION INSTABILITY IN BIREFRINGENT FIBERS
}

\author{
Stefano Trillo, S. Wabnitz
}

\section{To cite this version:}

Stefano Trillo, S. Wabnitz. MODULATIONAL POLARIZATION INSTABILITY IN BIREFRINGENT FIBERS. Journal de Physique Colloques, 1988, 49 (C2), pp.C2-301-C2-304. 10.1051/jphyscol:1988270 . jpa-00227687

\section{HAL Id: jpa-00227687 https://hal.science/jpa-00227687}

Submitted on 1 Jan 1988

HAL is a multi-disciplinary open access archive for the deposit and dissemination of scientific research documents, whether they are published or not. The documents may come from teaching and research institutions in France or abroad, or from public or private research centers.
L'archive ouverte pluridisciplinaire HAL, est destinée au dépôt et à la diffusion de documents scientifiques de niveau recherche, publiés ou non, émanant des établissements d'enseignement et de recherche français ou étrangers, des laboratoires publics ou privés. 


\title{
MODULATIONAL POLARIZATION INSTABILITY IN BIREFRINGENT FIBERS
}

\author{
S. TRILLO and S. WABNITZ \\ Fondazione Ugo Bordoni, Viale Europa 190, I-00144 Roma, Italy
}

\begin{abstract}
We study the modulational stability of a light beam propagating inside a birefringent optical fiber. The instability conditions and the gain curves for sidebands, with polarization parallel or orthogonal to the carrier, are derived analytically. We present beam propagation simulations showing that the effect of the instability over long pulses in presence of a weak initial modulation, is the generation of trains of ultrashort pulses. This occurs both in the normal and anomalous dispersion regime, and for a pump linearly polarized along either the fast or the slow axis of the fiber.
\end{abstract}

\section{1 - INTRQDUCTION}

The fact that a wave propagating in a nonlinear dispersive medium is subject to instabilities with respect to weak modulations is well known and is of importance in different fields of application $/ 1,2 /$. Only recently, however, this modulational instability has been observed in the propagation of light inside optical fibers $/ 3 /$, and its potential for generating ultra-high-repetition-rate trains of pulses has been demonstrated in the anomalous dispersion regime $14 /$.

The effect of including both state of polarization and birefringence in the description of modulational instability in fibers was not considered before. In the steady-state, however, self-induced polarization changes and natural birefringence lead to spatial instabilities /5/ which may induce intensity or phase-controlled switching of an intense light beam between orthogonal polarization states $/ 6 /$. In the present work we extend previous theories by including dispersion, birefringence and nonlinearity in the description of pulse propagation in a fiber: this leads to predicting new modulational polarization instabilities, generated by the interplay between the steady-state instability $/ 5 /$ and the four-photon mixing interaction $/ 7 /$. An important result of our approach is that modulational polarization instabilities may also occur in the normal dispersion regime.

\section{2 - THEORY}

Consider the propagation in a birefringent fiber of the complex field

$\mathbf{E}=\left[E_{x}(z, t) \exp \left\{i k_{x} z\right\} x+E_{y}(z, t) \exp \left\{i k_{y} z\right\} y\right] f(x, y) \exp \left\{-i \omega_{0} t\right\}$

where $f$ is the common transverse distribution of the two orthogonally polarized modes, with linear propagation constants $\mathrm{k}_{\mathrm{x}, \mathrm{y}}$. The two modes are mixed by the nonlinear displacement

$\mathbf{D}_{\mathrm{NL}}=\varepsilon_{0} \chi\left[\left(\mathbf{E} \cdot \mathbf{E}^{*}\right) \mathbf{E}+(1 / 2)(\mathbf{E} \cdot \mathbf{E}) \mathbf{E}^{*}\right]$

and the resulting coupled nonlinear equations for the (suitably normalized) amplitudes of the modes linearly polarized along the $\mathrm{x}$ and $\mathrm{y}$ directions, say $\mathrm{u}$ and $\mathrm{v}$ respectively, read

$$
\begin{aligned}
& i \partial u / \partial Z \pm(1 / 2) \partial^{2} u / \partial \tau^{2}+\kappa u+\left(|u|^{2}+(2 / 3)|v|^{2}\right) u+(1 / 3) u^{*} v^{2}=0 \\
& i \partial v / \partial Z \pm(1 / 2) \partial^{2} v / \partial \tau^{2}-\kappa v+\left(|v|^{2}+(2 / 3)|u|^{2}\right) v+(1 / 3) v^{*} u^{2}=0,
\end{aligned}
$$


where $+(-)$ holds for the anomalous (normal) dispersion regime, $\kappa \equiv\left(\mathrm{k}_{\mathrm{x}}-\mathrm{k}_{\mathrm{y}}\right) \mathrm{z}_{0} / 2, \mathrm{z}_{0}$ a characteristic distance $18 /$, and $Z \equiv z / z_{0}$. Note that, in writing eqs.(3), we implicitely assumed equal group velocities for pulses propagating in different modes. Equations (3) admit of the steady-state solution

$u^{0}=\exp \{i(\kappa+1) Z\}, v^{0}=0$,

corresponding to a linearly polarized pulse, whose polarization is aligned with either the slow or fast axis of the fiber, depending on the positive or negative sign of $\kappa$, respectively. Consider the stability of the solution (4) with respect a weak modulation. The perturbed version of eq.(4) is written as usual as

$u=(1+a) \exp \{i(\kappa+1) Z\}, v=b \exp \{i(\kappa+1) Z\}$, with

$a=A_{1} \exp \{i \lambda\}+A_{2} \exp \{-i \lambda\}, b=B_{1} \exp \{i \lambda\}+B_{2} \exp \{-i \lambda\}$, and $\lambda \equiv K Z-\Omega \tau$.

By inserting the expressions (5) into eqs.(3), one obtains linearized equations for the sidebands $a$ and $b$

$i \partial \mathrm{a} / \partial \mathrm{Z} \pm(1 / 2) \partial^{2} \mathrm{a} / \partial \tau^{2}+\mathrm{a}+\mathrm{a}^{*}=0$

$\mathrm{i} \partial \mathrm{b} / \partial \mathrm{Z} \pm(1 / 2) \partial^{2} \mathrm{~b} / \partial \tau^{2}-2 \mathrm{~kb}+(1 / 3)\left(\mathrm{b}^{*}-\mathrm{b}\right)=0$

Equation (6a), for sidebands with polarization parallel to the pump, coincides with the equation that one obtains in a purely scalar treatment, involving a single nonlinear Schroedinger equation $/ 8 /$. On the other hand, equation (6b) describes the early stage of the evolution of sidebands with linear polarization orthogonal to the carrier, and is peculiar to the present vectorial approach. The dispersion relationship associated with eq. (6b) is

$K^{2}(\Omega)=\left[ \pm(1 / 2) \Omega^{2}+2 \kappa\right]\left[ \pm(1 / 2) \Omega^{2}+2 \kappa+2 / 3\right]$

Detunings $\Omega$ yielding complex values of $\mathrm{K}$ lead to an exponential growth of the sidebands with fiber length $\mathrm{Z}$. From eq.(7), one immediately obtains analytical expressions for the growth rate, or gain, of this modulational instability. The gain, defined as $\mathrm{g} \equiv 2 \operatorname{Im}\{\mathrm{K}\}$, may be evaluated for the different cases of normal and anomalous dispersion, and for a pump on either the fast $(\kappa<0)$ or slow $(\kappa>0)$ axis. For example, figure 1(a) shows several gain curves, for different values of the parameter $p \equiv 1 / 3|\kappa|$, or normalized input power, for a typical set of fiber parameters $/ 9 /$, a pump polarized on the fast axis, and in the normal dispersion regime. As can be seen,
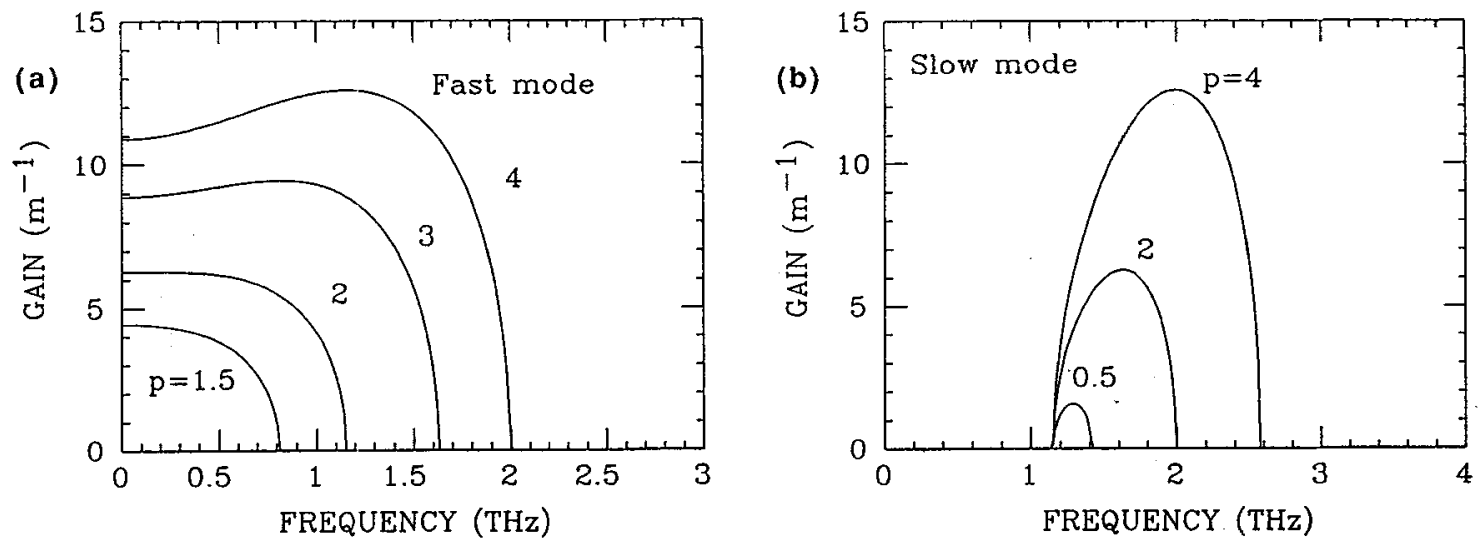

Fig, 1 - Gain of spatial growth of sidebands, which propagate in the normal dispersion regime, and are (a): linearly polarized along the slow axis, with a pump traveling in the fast mode, or (b) linearly polarized along the fast axis, with a pump in the slow mode. 
modulational instability first occurs as p grows up to unity (this is the bifurcation power of the continuous wave instability). Whenever $1 \leq \mathrm{p} \leq 2$, peak gain occurs for zero detuning, indicating that the spatial instability of the pump itself prevails. As soon as $\mathrm{p}>2$, the peak gain shifts to a finite detuning; this value is given, in general, by

$\pm \Omega^{2}=(2 / 3)+4 K$.

The above expression turns out to be precisely the phase matching condition $\mathbf{k}\left(\omega_{0}+\Omega\right)+\mathbf{k}\left(\omega_{0}-\Omega\right)=2 \mathbf{k}\left(\omega_{0}\right)$ for the four-photon mixing interaction between the intense pump and two orthogonally polarized sidebands. Fig. 2 (b) refers to the case of slow mode excitation; the peak gain is again given by eq. (8). The comparison between Figures 1 (a) and (b) shows that the continuous wave instability of the fast axis considerably broadens the gain curves, and prevents any modulational instability for $\mathrm{p}<1$. Conversely, the slow axis, which is stable in the steady state, does not exhibit a threshold in its modulational instability. Furthermore, it is remarkable that instability for the orthogonally polarized sidebands occurs both in the normal and anomalous regime, whereas the scalar instability described by equation 6(a) requires the anomalous dispersion regime.

\section{3 - ULTRASHORT PULSE GENERATION}

As proposed by Hasegawa /10/ and recently experimentally demonstrated /4/, modulational instabilities have potential for generating trains of ultrashort pulses at the output of a fiber, when a weakly modulated beam is launched the at the fiber input. The initial modulation of the carrier may be obtained for example by means of a tunable semiconductor laser: in this case the instability is induced at a given frequency detuning which in turn fixes the repetition rate of the generated train of pulses. These earlier results were based on the scalar treatment (i.e., eq.6(a)), predicting modulational instability in the anomalous dispersion regime only. The present approach, which includes the state of polarization and fiber birefringence in the description, shows that modulational instabilities exist and can be exploited also in the normal dispersion regime. We investigated this point by means of the beam propagation method, which allows for studying the evolution of a weakly modulated wavetrain over distances that lie beyond the limit of validity of the linearized eqs.(6).

Figures 2 (a) and (b) show examples of the coupling of light between the slow and fast modes with amplitudes $u$ and $v$, respectively, in the normal dispersion regime. In fig. 2(a), a relatively long square pulse whose normalized peak power is $\mathrm{p}=3$ is coupled into the fast mode at the fiber input, while a weak modulation is imposed onto the orthogonal axis. As can be seen, the continuous-wave spatial instability leads to abrupt transfers of energy between the modes, until the dispersive broadening introduces a severe distortion and beakup into an irregular sub-pulse structure. It is worth noting that, in this case, the low-frequency instability prevented the development of the induced instability at the modulation frequency. On the other hand, fig. 2(b) shows that when the input wave (with $p=4 / 3$ ) is coupled into the slow mode, the modulation initially present in the orthogonal polarization grows with distance, eventually leading to a sequence of short pulses, which propagate unchanged until heavy depletion and broadening of the carrier waveform occurs. Figures 3(a) and (b) display sequences of pulses generated by modulational polarization instabilities, in the normal and anomalous dispersion regimes, respectively. Fig. 3(a) shows the field component propagating in the fast axis, as extracted from fig. $2(\mathrm{~b})$ with $Z=2.56$. On the other hand, fig. 3(b) shows an example of a soliton-like train of pulses, which is generated in the polarization orthogonal to the pump $(p=4 / 3)$ and in the anomalous dispersion regime.

\section{4 - CONCLUSIONS}

We found that, if the unavoidable changes in the state of polarization along a fiber are accounted for, modulational instabilities may occur both in the normal and in the anomalous dispersion regime. Waves that are spatially unstable in the steady-state will ultimately become stable with respect to sufficiently rapid modulations: the time scale is fixed by group-velocity dispersion. Conversely, a spatially stable uniform wavetrain, such as for example one which is linearly polarized along the slow axis, may decay into orthogonally polarized sidebands whose detuning falls in a certain range of frequencies, which are in turn determined by dispersion and birefringence.

These phenomena should make possible the generation of ultrashort trains of pulses in the normal dispersion regime. On the other hand, since all-optical fiber switches are based on the continuous wave spatial instability, the present study shows that when short pulses are involved the instability gain eventually decreases, indicating that a fundamental limit (set by dispersion) exists to the ultimate speed of these devices. Finally, as discussed in detail elsewhere $/ 11 /$, modulational polarization instabilities offer new possibilities for generating squeezed light.

\section{REFERENCES}

/1/ V.I. Bespalov and V.I. Talanov, JETP Lett. 3, 307 (1966).

12/ T.J. Benjamin and J.E. Feir, J. Fluid Mech. 27, 417 (1967). 
/3/ K. Tai, A. Hasegawa, and A. Tomita, Phys. Rev. Lett. 56, 135 (1986)

/4/ K. Tai, A. Tomita, J.L. Jewell, and A. Hasegawa, Appl. Phys. Lett. 49, 236 (1986).

15/ B. Daino, G. Gregori, and S. Wabnitz, J. Appl. Phys. 58, 4512 (1985); G. Gregori and S. Wabnitz, Phys. Rev. Lett. 56, 600 (1986); H.G. Winful, Opt. Lett. 11, 33 (1986).

16/ S. Trillo, S. Wabnitz, R.H. Stolen, G. Assanto, C.T. Seaton, and G.I. Stegeman, Appl. Phys. Lett. 49, 1224 (1986).

7/ R.H. Stolen and J.E. Bjorkholm, IEEE J. Quantum Electron. QE-18, 1062 (1982).

/8/ L.F. Mollenauer, R.H. Stolen, and J.P. Gordon, Phys. Rev. Lett. 45, 1095 (1980)

19/ S. Wabnitz, to be published on Phys. Rev. A (1988).

/10/ A. Hasegawa, Opt. Lett. 9, 288 (1984).

/11/ T.A.B. Kennedy, and S. Wabnitz, to be published on Phys. Rev. A (1988).
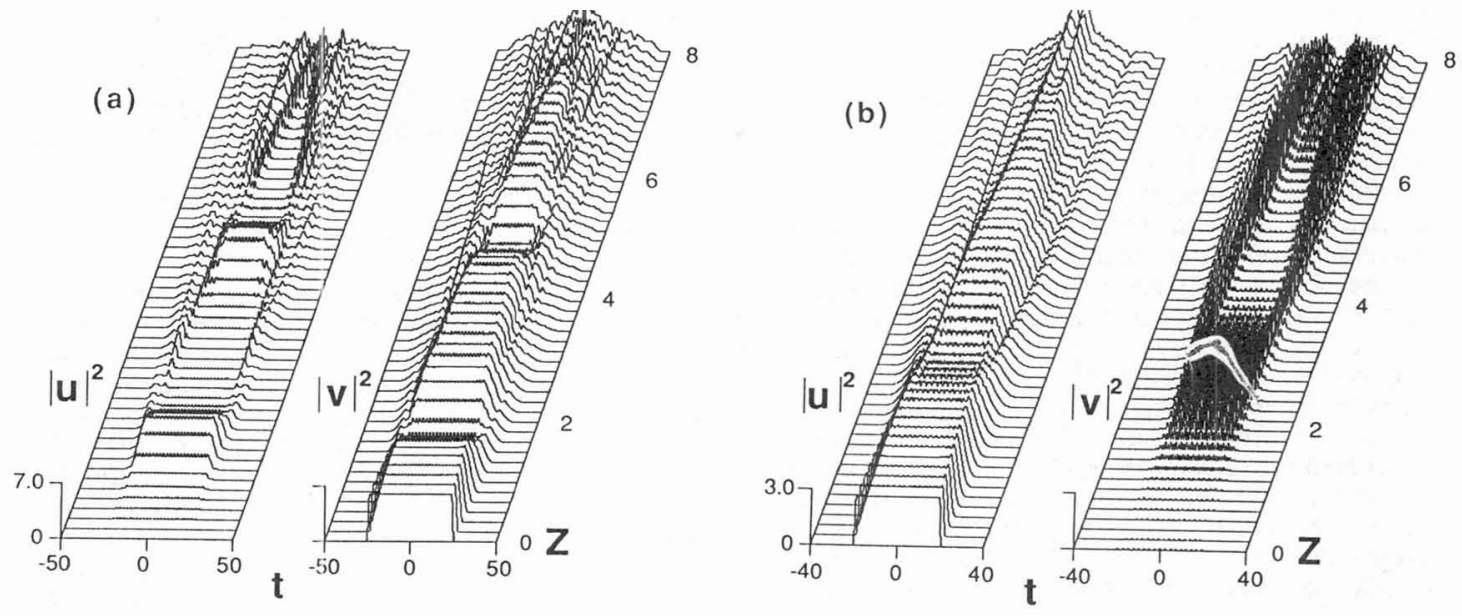

Fig. 2 - Evolution of light propagating in the two orthogonally polarized modes, in a frame of reference traveling with the common group velocity, and in the normal dispersion regime. (a): input pulse (with $p=3$ ) in the fast mode, and modulation on the orthogonal axis; $(b)$ : input pulse $(p=4 / 3)$ in the slow mode.
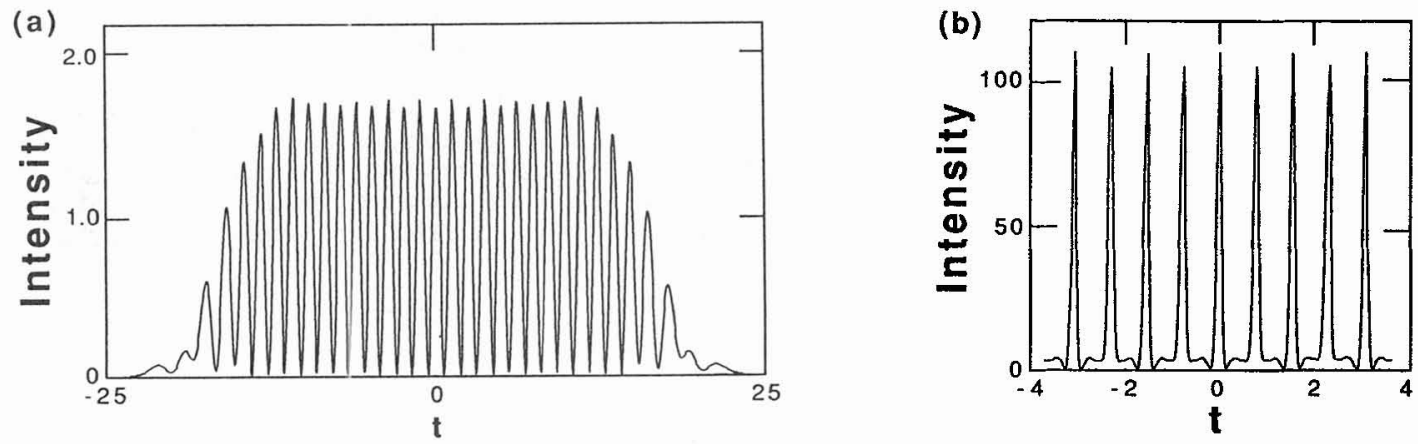

Fig. 3 - (a) Train of pulses emerging from an analyzer placed at the fiber output and oriented along the fast axis, for a fiber of length $Z=2.56$, in the same conditions as in fig. 2(b); (b) same as in (a), but with a carrier in the fast axis, the analyzer along the slow axis and in the anomalous dispersion regime. 